Supporting Information:

\title{
A Stochastic Regression Approach to Analyzing Thermodynamic Uncertainty in Chemical Speciation Modeling
}

Christopher L. Weber, Jeanne M. VanBriesen* and Mitchell S. Small

Department of Civil and Environmental Engineering, Carnegie Mellon University, Pittsburgh, PA 15213

*Corresponding author email: jeanne@cmu.edu

(24 pages, 2 Tables, 18 Figures) 
Supporting Information-A Stochastic Regression Approach to Analyzing Thermodynamic Uncertainty in Chemical Speciation Modeling

\section{Temperature Uncertainty Analysis}

Early in the modeling work, the assumption was made that eliminating all data outside of the $20^{\circ} \mathrm{C}-30^{\circ} \mathrm{C}$ range would make temperature correction uncertainty relatively small in comparison to the uncertainty in ionic strength correction. To validate this assumption, a modified MCMC regression model was created where the change in enthalpy value for the formation reaction of interest $\left(\Delta \mathrm{H}_{\mathrm{rxn}}\right)$ was treated as uncertain. In the original MCMC model, the minor temperature corrections from $20^{\circ} \mathrm{C}$ or $30^{\circ} \mathrm{C}$ to $25^{\circ} \mathrm{C}$ were made with the Van't Hoff equation, shown as equation SI1 below. The change in enthalpy was assumed fixed at the value given in the Visual MINTEQ database. To run the uncertainty analysis, all $\Delta \mathrm{H}_{\mathrm{rxn}}$ 's were allowed to vary over a normal distribution with standard deviation of $5 \mathrm{~kJ} / \mathrm{mol}$, which was an average uncertainty value in the estimates presented in the JESS thermodynamic database. To make sure these uncertainties remained unchanged through Bayesian updating, the cut function in WinBUGS (see Data Reduction and Combination Details section) was utilized.

Equation SI-1: $\ln \left(\frac{\left.K\right|_{T_{2}}}{\left.K\right|_{T_{1}}}\right)=\frac{\Delta H_{r \times n}}{R}\left(\frac{1}{T_{1}}-\frac{1}{T_{2}}\right)$

The assumption was verified by the results of this revised model, which are shown in the full results table (Table SI-1) on page 6 of Supporting Information for each species. As is evident in Table SI-1, the results with an uncertain enthalpy of reaction were extremely close to the results with a fixed enthalpy of reaction. This suggests that temperature correction uncertainty, at least over the range of $20^{\circ} \mathrm{C}-30^{\circ} \mathrm{C}$, is relatively small compared to ionic strength extrapolation uncertainty. 
Supporting Information-A Stochastic Regression Approach to Analyzing Thermodynamic Uncertainty in Chemical Speciation Modeling

\section{Data Reduction and Combination Details}

Because the data for secondary species formation is given in several different forms in the JESS database, a series of assumptions and combinations was necessary to make use of all data. The first such simplification occurred out of necessity to achieve model convergence. Since all reactions of interest in the model involved the EDTA ${ }^{-4}$ species, each simultaneous regression fit a different value for the $b_{i}$ parameter for this species. Convergence of the model was thus extremely difficult to achieve. Therefore, the $b_{i}$ value for the $\left[\mathrm{EDTA}^{-4}\right]$ species was assumed equal to zero, since any speciesspecific $b_{i}$ value (e.g., $b_{i}$ for MEDTA) acts to absorb the fitted value of $b_{i, E D T A-4}$ in an overall reaction summation of $b_{i}$ values.

One other similar problem with modeling the data was that for the secondary complexes-MHL, MOHL, and $\mathrm{M}(\mathrm{OH})_{2} \mathrm{~L}$ - each species' overall formation reaction could be constructed from several different reaction types cited in the literature, most of which were dependent on primary complex formation constants. For example, for the species $\mathrm{Fe}(\mathrm{OH}) \mathrm{EDTA}^{-2}$, data points were distributed between reaction types as follows:

(1) $\mathrm{Fe}^{+3}+\mathrm{OH}^{-1}+\mathrm{EDTA}^{-4}=\mathrm{Fe}(\mathrm{OH}) \mathrm{EDTA}^{-2} \quad 1$ data value

(2) $\mathrm{FeEDTA}^{-1}+\mathrm{OH}^{-1}=\mathrm{Fe}(\mathrm{OH}) \mathrm{EDTA}^{-2} \quad 3$ data values

(3) $\mathrm{FeEDTA}^{-1}+\mathrm{H}_{2} \mathrm{O}=\mathrm{Fe}(\mathrm{OH}) \mathrm{EDTA}^{-2}+\mathrm{H}^{+1} \quad 8$ data values

To fit all of these data simultaneously, simple reaction arithmetic, dependent on the primary (ML) reaction constants, was performed until all data were in the same, overall formation reaction form. By including the primary reaction coefficients in these secondary reaction regressions, however, the chance of these relatively less precise data 
Supporting Information-A Stochastic Regression Approach to Analyzing Thermodynamic Uncertainty in Chemical Speciation Modeling

'informing' the relatively more precise primary reaction coefficients $\left(\beta_{\mathrm{HL}}^{\mathrm{o}}\right.$ or $\left.\beta_{\mathrm{ML}}^{\mathrm{o}}\right)$ was introduced. This problem was managed by using the internal 'cut' function in WinBUGS (1) to stop the flow of information from secondary (MHL, $\mathrm{M}(\mathrm{OH}) \mathrm{L})$ to primary data. According to the WinBUGS manual, this function acts "as a valve in the graph—prior information is allowed to flow 'downwards' through the cut, but likelihood information is prevented from flowing upwards." (1) This 'cut' and its use in the construction of the overall secondary constants is illustrated in Figure SI-1 below.

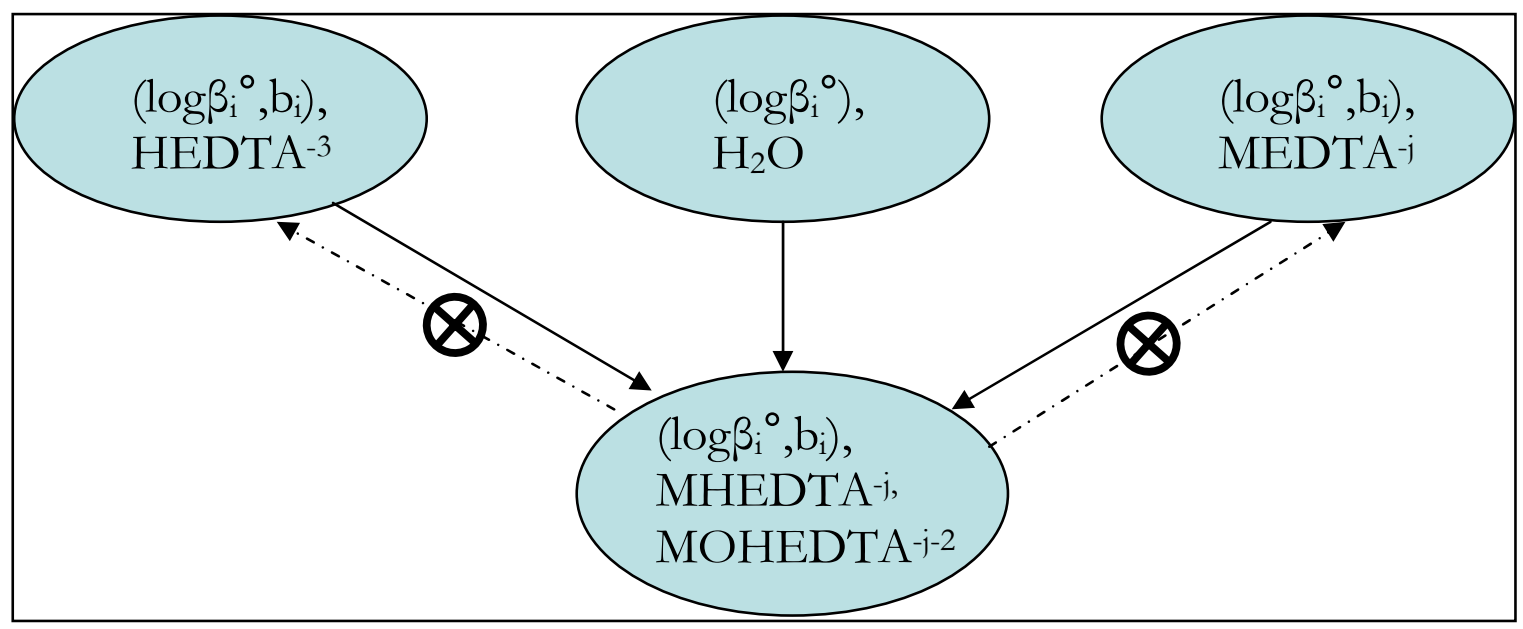

Figure S1: Depiction of statistical model for thermodynamic constants. Arrows depict information flow contained in the likelihood updating of the model, and the x's depict where information flow was 'cut'.

\section{Modeled Chemical System Details}

The detailed model natural water is shown below, with all concentrations in Molarity.

These concentrations were calculated from average values given in the EPA Storet database for Allegheny County, PA from the years 2003 to 2005. The EDTA

concentration is a typical value for natural streams taken from (2). Below the aqueous species present in the model, the solid species that were allowed to precipitate in the solids case are given. 


\section{Full Statistical Model Results}

Table S1: Results of the Stochastic Regression MCMC model. The first four columns show the result of the full model, the next four columns show the results of the temperature uncertainty analysis, the next four show the values listed in the JESS and Visual

MINTEQ databases for comparison, and the final four columns show the skewness coefficients and kurtosis for the full model. For the secondary constants, $\left(\beta_{\mathrm{MHL}}^{\circ}, \beta^{\circ}{ }_{\mathrm{MOHL}}, \beta^{\circ} \mathrm{M}(\mathrm{OH}) 2 \mathrm{~L}\right)$ more than one value is shown for the JESS program, because the overall formation reaction can be created by reaction arithmetic in a variety of ways. Components with a *** designation were included stochastically in the statistically model but deterministically in the speciation modeling.

\begin{tabular}{|c|c|c|c|c|c|c|c|c|c|c|c|c|c|c|c|c|}
\hline & $\log \beta^{\mathrm{O}}$ mean & $\log \beta^{0} \sigma$ & $b_{i}$ mean & $b_{i} \sigma$ & $\log \beta^{0} \mu_{\mathrm{HS}}$ & $\log \beta^{0} \sigma_{H S}$ & $b_{i} \mu_{H S}$ & $b_{i} \sigma_{H S}$ & JESS & JESS & JESS & VMINT & Skew $\beta$ o & Kurt $\beta o$ & Skew $b_{i}$ & Kurt $b_{i}$ \\
\hline HEDTA-3 & 11.03 & 0.04 & -0.47 & 0.10 & 11.03 & 0.11 & 11.03 & 0.04 & 10.61 & - & - & 10.95 & 0.04 & 0.33 & -0.05 & -0.05 \\
\hline H2EDTA-2 $* * *$ & 17.75 & 0.04 & 20.92 & 0.04 & 17.75 & 0.04 & 20.92 & 0.04 & 17.68 & 17.75 & - & 17.22 & 0.10 & -0.10 & 0.07 & -0.28 \\
\hline H3EDTA-1 *** & 20.92 & 0.04 & -1.17 & 0.09 & 20.92 & 0.04 & -1.17 & 0.09 & 19.92 & 20.92 & - & 20.34 & -0.10 & -0.16 & -0.1 & -0.16 \\
\hline $\operatorname{H} 4 \operatorname{EDTA}(\mathrm{aq}) * * *$ & 23.13 & 0.04 & -1.4 & 0.09 & 23.14 & 0.04 & -1.4 & 0.1 & 23.19 & 23.13 & - & 22.55 & -0.07 & -0.36 & -0.06 & -0.05 \\
\hline CuEDTA-2 & 20.29 & 0.17 & -2.15 & 1.43 & 20.29 & 0.18 & -2.11 & 1.43 & 20.36 & - & - & 20.49 & 0.02 & 1.06 & 0.09 & 0.64 \\
\hline CuHEDTA-1 & 23.67 & 0.22 & -2.28 & 1.38 & 23.68 & 0.22 & -2.22 & 1.38 & 23.9 & 23.87 & 23.46 & 24.02 & 0.07 & 0.06 & 0.09 & 0.43 \\
\hline CuOHEDTA-3 & 22.50 & 0.16 & -1.61 & 1.39 & 22.49 & 0.17 & -1.57 & 1.39 & 22.39 & 22.44 & 22.49 & 22.44 & 0.06 & 0.86 & 0.08 & 0.50 \\
\hline ZnEDTA-2 & 18.15 & 0.09 & -0.47 & 0.62 & 18.15 & 0.09 & -0.46 & 0.58 & 18.15 & - & - & 18 & 0.17 & 0.83 & 0.21 & 0.91 \\
\hline ZnHEDTA-1 & 21.49 & 0.09 & -0.85 & 0.61 & 21.50 & 0.08 & -0.84 & 0.56 & 21.7 & 21.56 & 21.3 & 21.43 & 0.11 & 0.83 & 0.21 & 1.24 \\
\hline ZnOHEDTA-3 & 20.00 & 0.17 & 0.13 & 0.63 & 19.99 & 0.20 & 0.12 & 0.63 & 19.9 & 19.83 & 19.84 & 19.76 & 0.33 & 5.30 & 0.07 & 0.79 \\
\hline NiEDTA-2 & 20.13 & 0.07 & -1.04 & 0.20 & 20.13 & 0.07 & -1.03 & 0.20 & 20.13 & - & - & 20.11 & -0.05 & 0.83 & 0.16 & 0.71 \\
\hline NiHEDTA-1 & 23.79 & 0.11 & -1.09 & 0.22 & 23.79 & 0.11 & -1.08 & 0.21 & 24 & 23.68 & - & 23.64 & 0.31 & 1.63 & 0.10 & 0.51 \\
\hline NiOHEDTA-3 & 21.78 & 0.18 & 0.52 & 0.34 & 21.78 & 0.19 & 0.51 & 0.34 & 21.8 & 21.68 & - & 21.56 & 0.05 & 1.73 & 0.04 & 1.23 \\
\hline CaEDTA-2 & 12.22 & 0.11 & -0.87 & 0.38 & 12.22 & 0.11 & -0.85 & 0.38 & 12.38 & & - & 12.44 & 0.07 & 0.18 & -0.10 & 0.20 \\
\hline CaHEDTA-1 & 15.71 & 0.15 & -1.92 & 0.60 & 15.71 & 0.15 & -1.89 & 0.60 & 16 & 15.47 & - & 15.97 & 0.04 & 4.46 & 0.40 & 2.66 \\
\hline MnEDTA-2 & 15.55 & 0.14 & -0.85 & 1.31 & 15.55 & 0.14 & -0.85 & 1.32 & 15.38 & - & - & 15.6 & -0.01 & 0.74 & -0.12 & 1.28 \\
\hline MnHEDTA-1 & 19.11 & 0.15 & -0.31 & 1.56 & 19.11 & 0.18 & -0.28 & 1.93 & 19.1 & 18.89 & 19.17 & 19.13 & 0.49 & 2.21 & 0.16 & 1.51 \\
\hline MgEDTA-2 & 10.14 & 0.21 & -2.03 & 0.93 & 10.12 & 0.20 & -2.12 & 0.91 & 10.27 & - & - & 10.58 & 0.04 & 0.94 & -0.04 & 1.01 \\
\hline MgHEDTA-1 & 15.09 & 0.17 & 4.24 & 1.73 & 15.09 & 0.17 & 4.28 & 1.76 & 15.1 & 14.54 & 14.56 & 15.01 & -0.50 & 2.79 & -0.65 & 3.67 \\
\hline PbEDTA-2 & 19.64 & 0.12 & -0.67 & 0.52 & 19.64 & 0.12 & -0.66 & 0.54 & 19.67 & - & - & 19.71 & -0.03 & 0.63 & 0.16 & 0.90 \\
\hline PbHEDTA-1 & 22.56 & 0.32 & -0.95 & 0.66 & 22.56 & 0.34 & -0.95 & 0.69 & 21.86 & 22.86 & 22.85 & 22.54 & 0.19 & 1.06 & 0.12 & 0.13 \\
\hline CoEDTA-2 & 18.00 & 0.18 & 0.07 & 1.62 & 18.01 & 0.18 & 0.12 & 1.58 & 17.94 & - & - & 18.16 & 0.02 & 0.42 & 0.01 & 0.23 \\
\hline CoHEDTA-1 & 21.43 & 0.22 & -0.46 & 1.21 & 21.43 & 0.20 & -0.43 & 1.19 & 21.34 & 21.38 & 21.5 & 21.59 & 0.66 & 3.62 & 0.13 & 0.39 \\
\hline AlEDTA-1 & 19.08 & 0.13 & 0.65 & 1.29 & 19.08 & 0.13 & 0.64 & 1.25 & 19.12 & - & - & 18.96 & 0.22 & 1.50 & 0.28 & 1.42 \\
\hline $\operatorname{AlHEDTA}(\mathrm{aq})$ & 21.82 & 0.25 & 0.58 & 1.56 & 21.82 & 0.24 & 0.61 & 1.55 & 21.6 & 21.93 & 21.29 & 21.78 & 0.28 & 2.28 & 0.06 & 1.04 \\
\hline AlOHEDTA-2 & 26.88 & 0.16 & 0.45 & 1.23 & 26.89 & 0.16 & 0.44 & 1.20 & 27.19 & 26.91 & 26.79 & 26.63 & 0.19 & 1.32 & 0.16 & 1.10 \\
\hline FeEDTA-1 & 27.49 & 0.20 & -1.01 & 0.98 & 27.50 & 0.20 & -1.03 & 0.95 & 27.52 & - & - & 27.66 & 0.01 & 0.55 & 0.18 & 0.59 \\
\hline FeHEDTA(aq) & 28.99 & 0.14 & -0.69 & 0.29 & 29.00 & 0.14 & -0.67 & 0.28 & 29.2 & 28.99 & 28.95 & 29.17 & 0.47 & 5.74 & 0.06 & 5.58 \\
\hline FeOHEDTA-2 & 33.83 & 0.18 & -0.64 & 0.96 & 33.83 & 0.18 & -0.66 & 0.93 & 33.8 & 33.82 & 34.32 & 33.84 & 0.02 & 0.40 & 0.18 & 0.59 \\
\hline
\end{tabular}




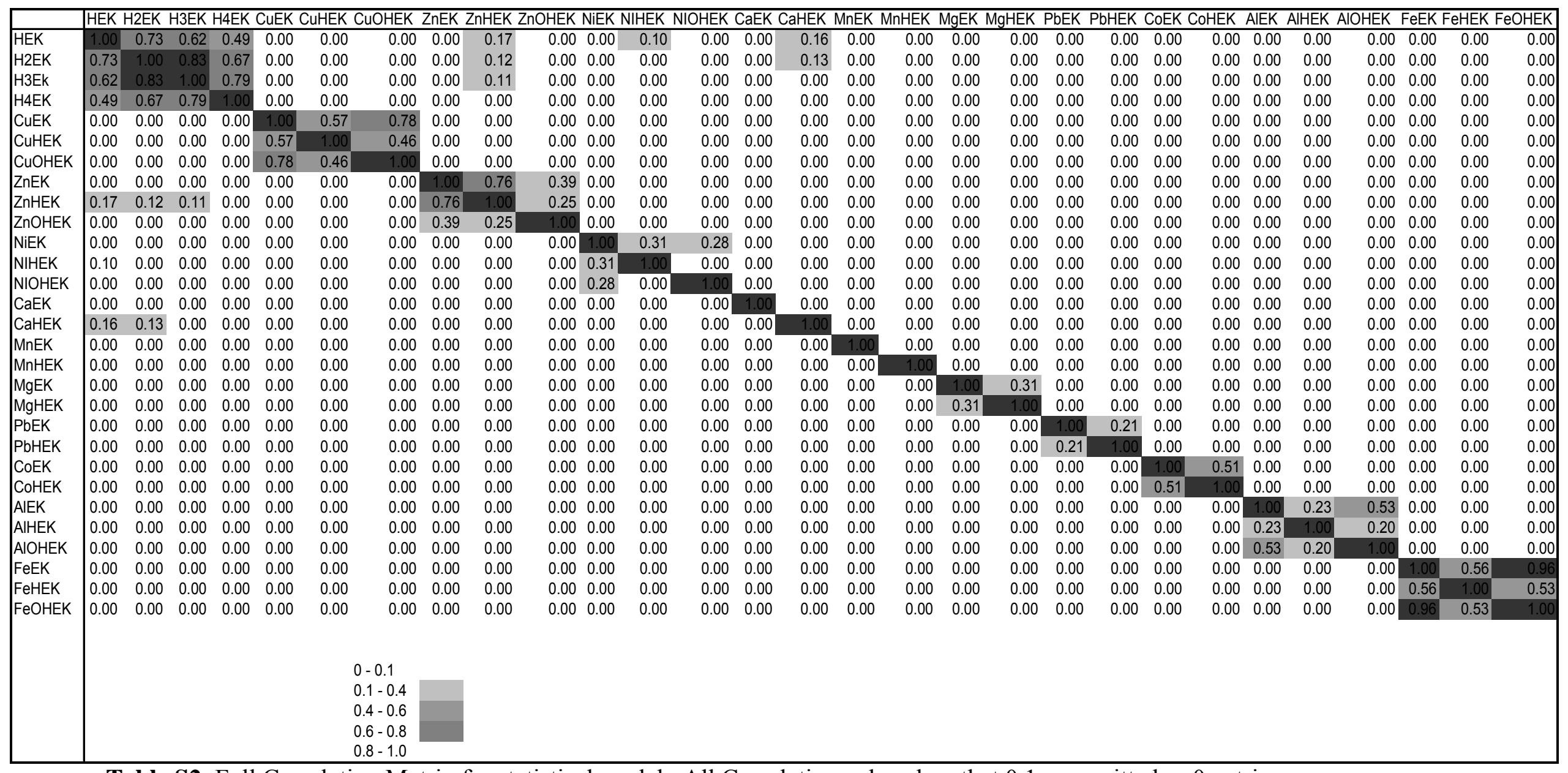

Table S2: Full Correlation Matrix for statistical model. All Correlation values less that 0.1 are omitted as 0 entries. 
Supporting Information-A Stochastic Regression Approach to Analyzing Thermodynamic Uncertainty in Chemical Speciation Modeling

\section{Effect of Uncertain EDTA Constants on Overall Metal Speciation}

As stated in the paper, it was known that varying the thermodynamic parameters for EDTA species would have an impact on the overall speciation of metals through the MEDTA species. A sample plot of this effect from the simulation with solids is shown in Figure SI-2 below, which shows the stochastic speciation of nickel for this system (panel a) and the distributions' skewness coefficients (b). It is evident from this plot of skewness vs. $\mathrm{pH}$ that all of the uncertainty in the calculated values of non-EDTA species is directly due to the uncertainty affiliated with the EDTA stability constants. This result verifies that in competitive equilibrium models, varying some uncertain inputs while leaving others constant will not produce any additional uncertainty through competition with deterministic parameters. This result allows the model user to choose which parameters are likely to be the most uncertain rather than requiring estimation of uncertainty for all parameters. 
Supporting Information-A Stochastic Regression Approach to Analyzing Thermodynamic Uncertainty in Chemical Speciation Modeling
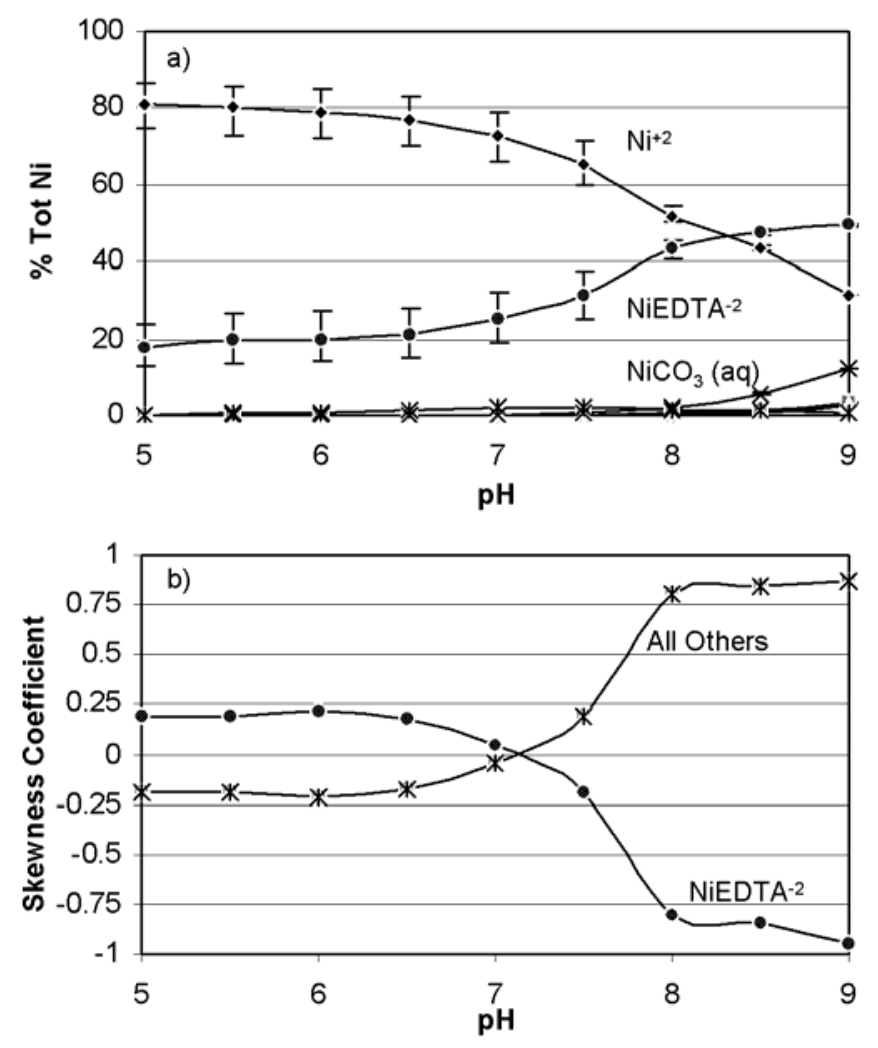

Figure S2: Calculated speciation of Nickel in the solids simulation and the skewness coefficient of the output distributions as a function of $\mathrm{pH}$. It is clear that all non-EDTA species are perfectly correlated and skewed precisely opposite of NiEDTA-2 . 
Supporting Information-A Stochastic Regression Approach to Analyzing Thermodynamic Uncertainty in Chemical Speciation Modeling

\section{Detailed Correlation Matrices}

While sample correlation coefficients were presented in Figure 4, relatively more information can be gathered by examining full correlation matrices of all the important species as a function of $\mathrm{pH}$. The following is a series of correlation matrices calculated for both the no solids and solids case. The correlation trends are much easier to see by examining these full matrices over the course of $\mathrm{pH}$. For example, as seen in Figure SI-3, the no solids series, the relative growth of NiEDTA $^{-2}$ is exhibited through its correlation with other species growing increasingly negative from $\mathrm{pH} 7$ to 9 . Similarly, the induced correlation structure of $\mathrm{FeEDTA}^{-1}$ and FeOHEDTA ${ }^{-2}$ can be seen easily in the higher $\mathrm{pH}$ 's, where it is the only strongly positively correlated pair due to its input correlation.

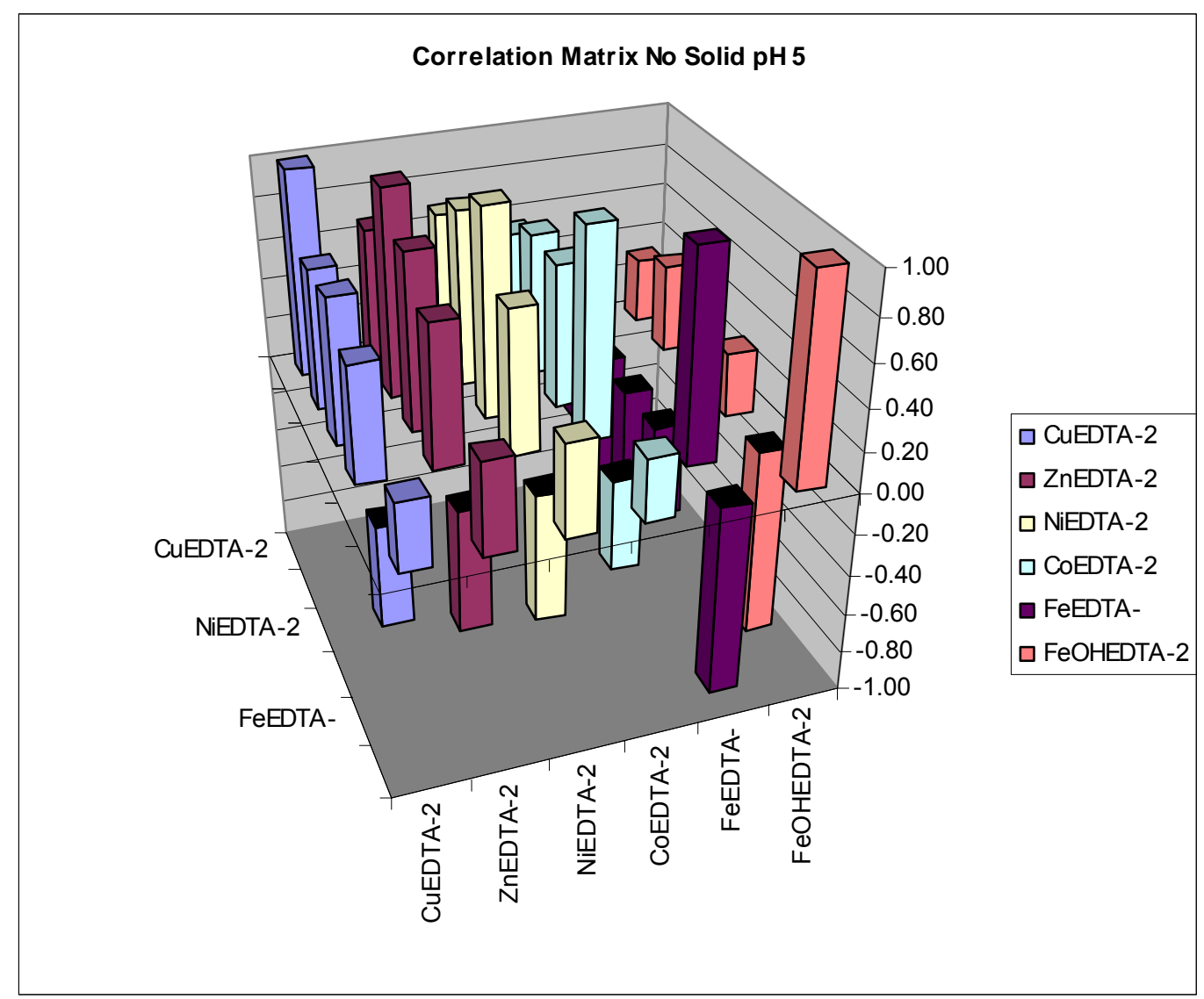

Figure S3: Correlation Matrix Series for No solids case, pH 5 
Supporting Information-A Stochastic Regression Approach to Analyzing Thermodynamic Uncertainty in Chemical Speciation Modeling

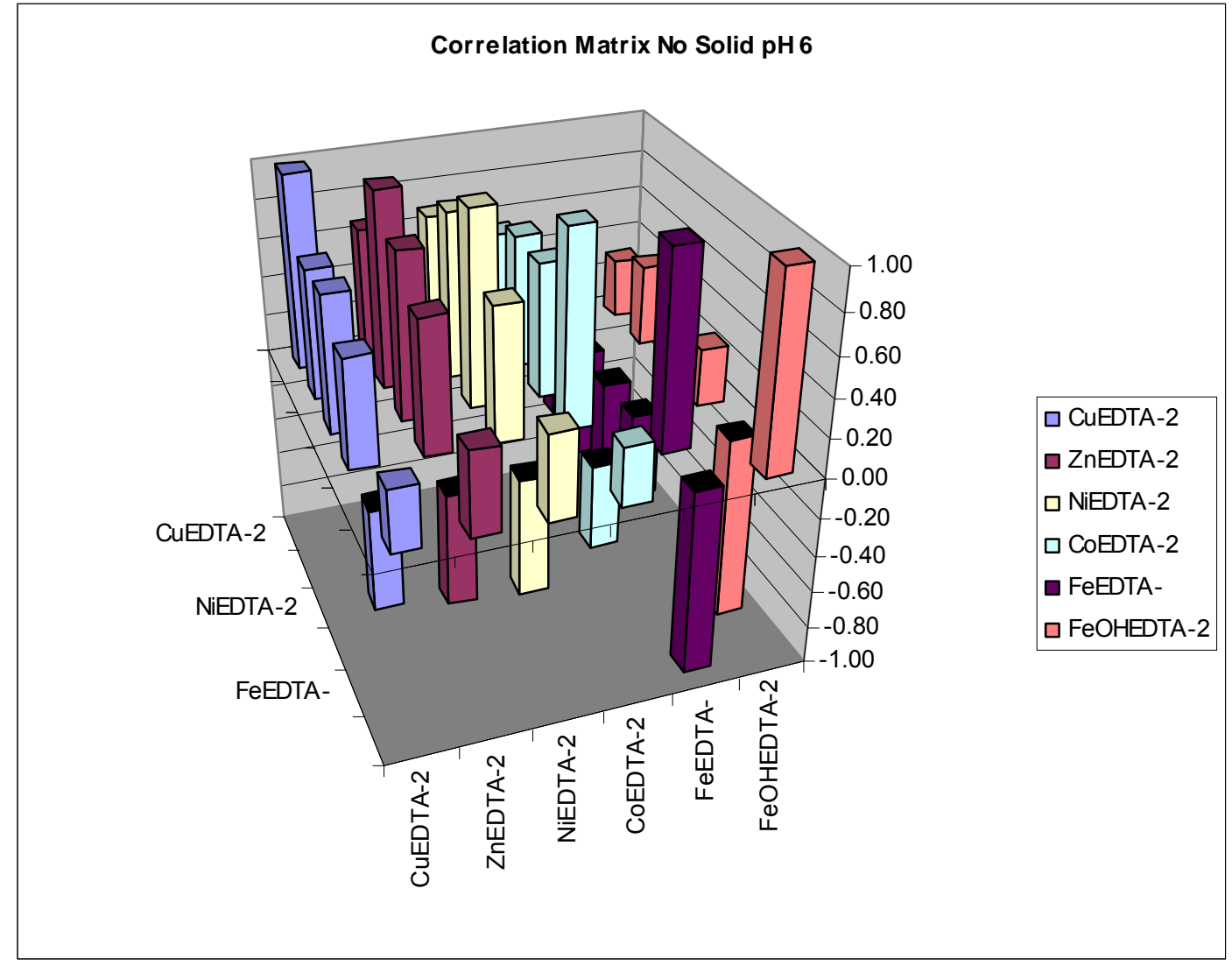

Figure S4: Correlation Matrix Series for No solids case, pH 6 
Supporting Information-A Stochastic Regression Approach to Analyzing Thermodynamic Uncertainty in Chemical Speciation Modeling

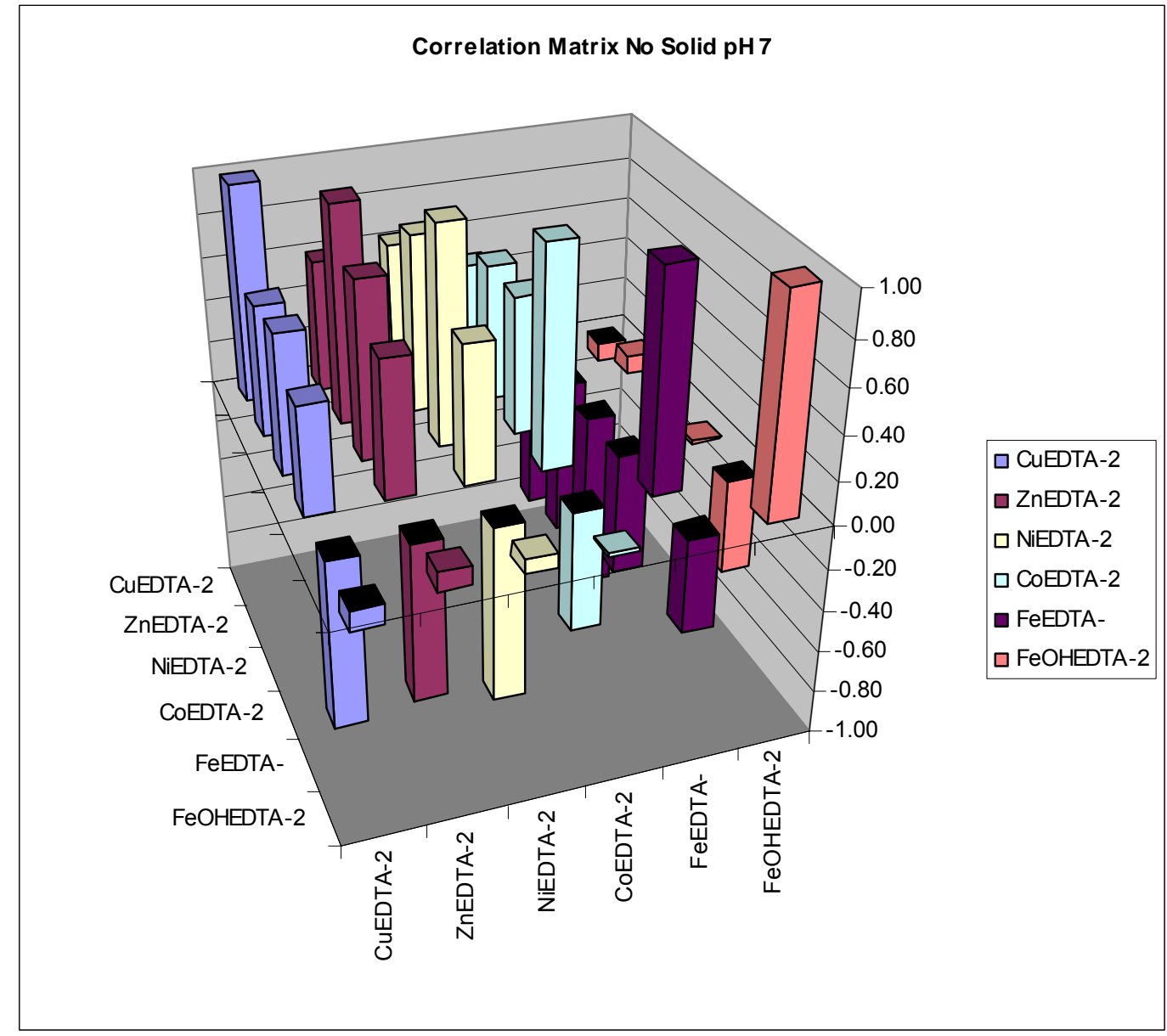

Figure S5: Correlation Matrix Series for No solids case, $\mathrm{pH} 7$ 
Supporting Information-A Stochastic Regression Approach to Analyzing Thermodynamic Uncertainty in Chemical Speciation Modeling

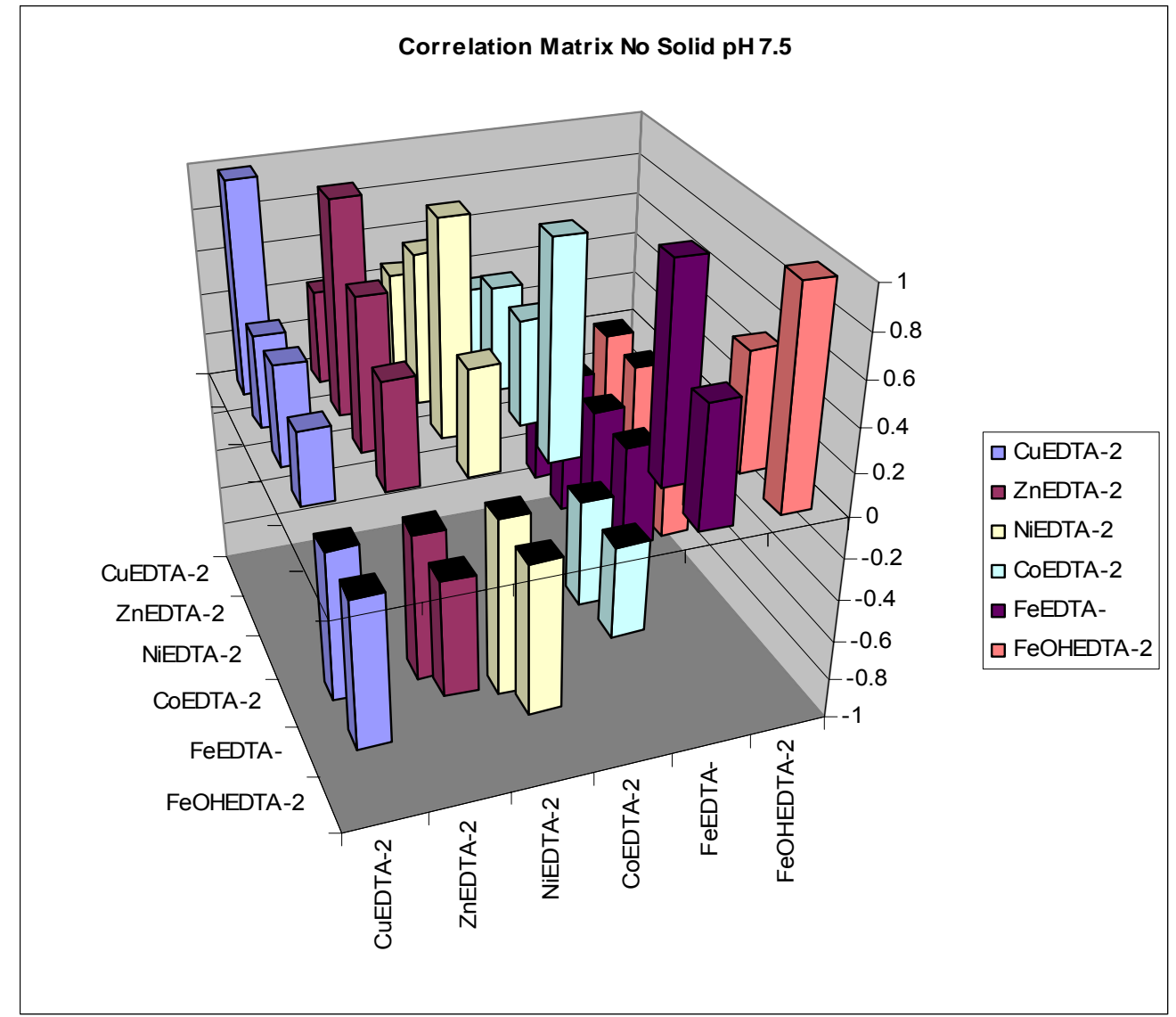

Figure S6: Correlation Matrix Series for No solids case, pH 7.5 
Supporting Information-A Stochastic Regression Approach to Analyzing Thermodynamic Uncertainty in Chemical Speciation Modeling

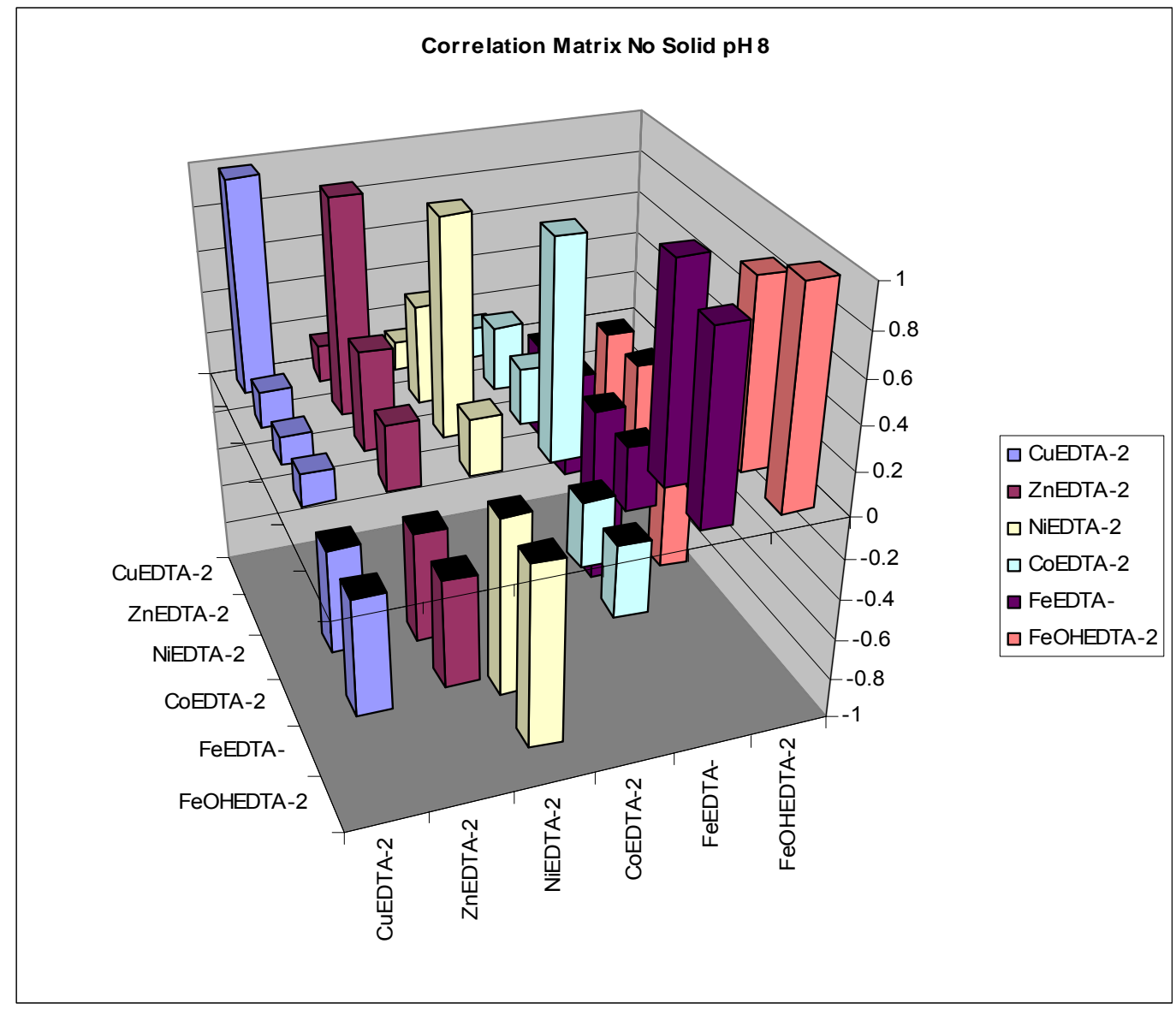

Figure S7: Correlation Matrix Series for No solids case, pH 8 
Supporting Information-A Stochastic Regression Approach to Analyzing Thermodynamic Uncertainty in Chemical Speciation Modeling

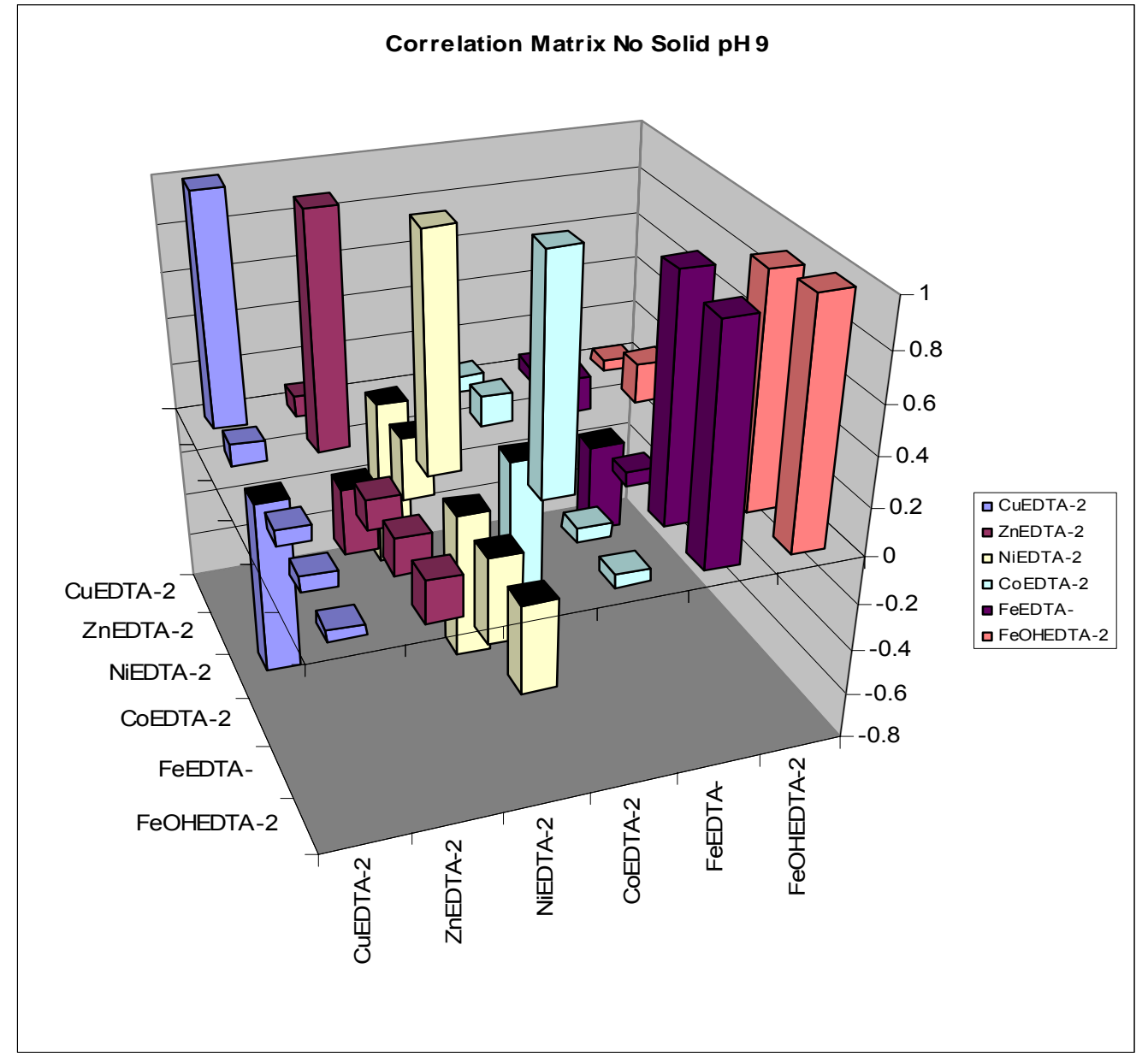

Figure S8: Correlation Matrix Series for No solids case, pH 9 
Supporting Information-A Stochastic Regression Approach to Analyzing Thermodynamic Uncertainty in Chemical Speciation Modeling

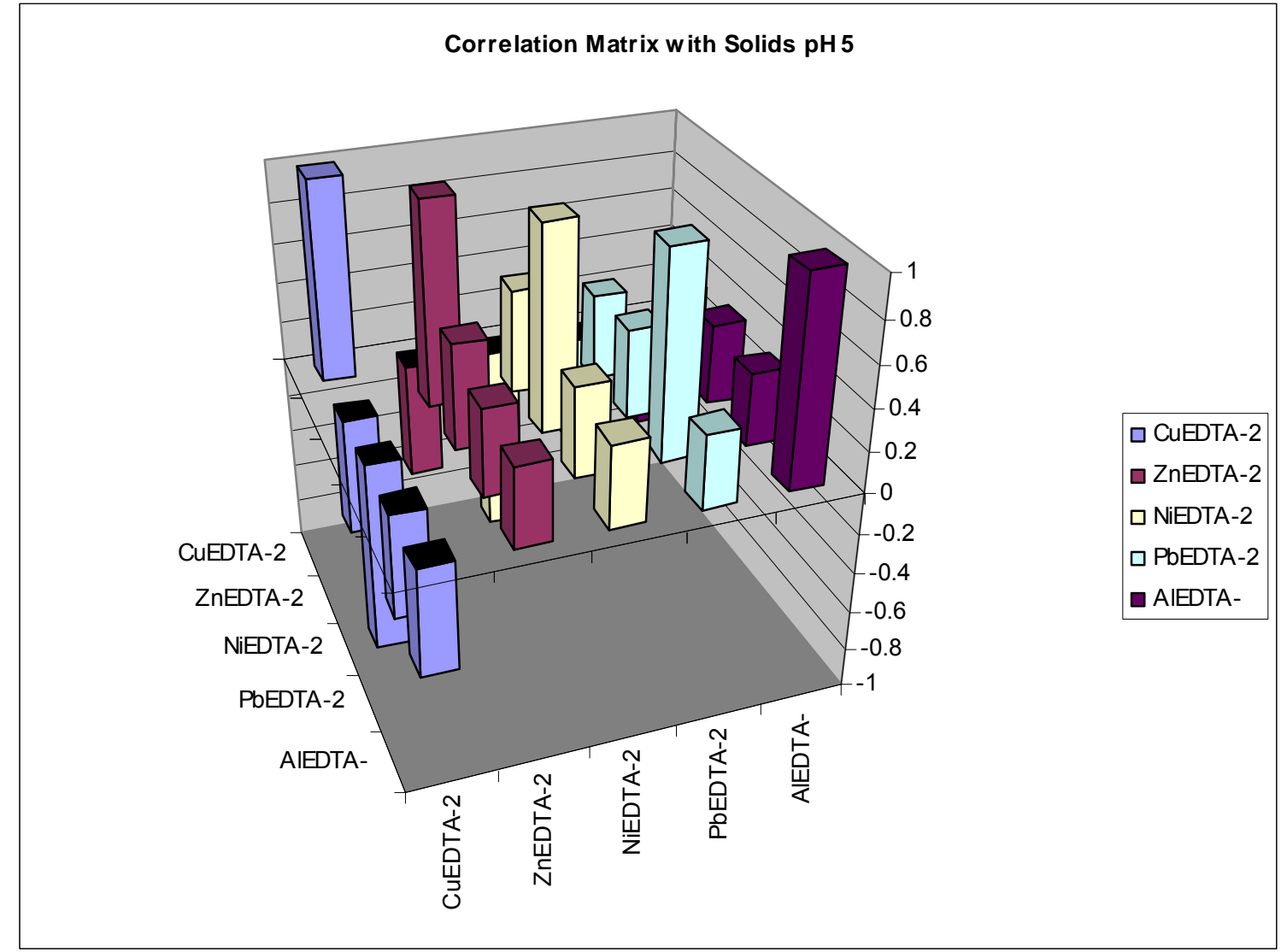

Figure S9: Correlation Matrix Series for Solids Case, pH 5 
Supporting Information-A Stochastic Regression Approach to Analyzing Thermodynamic Uncertainty in Chemical Speciation Modeling

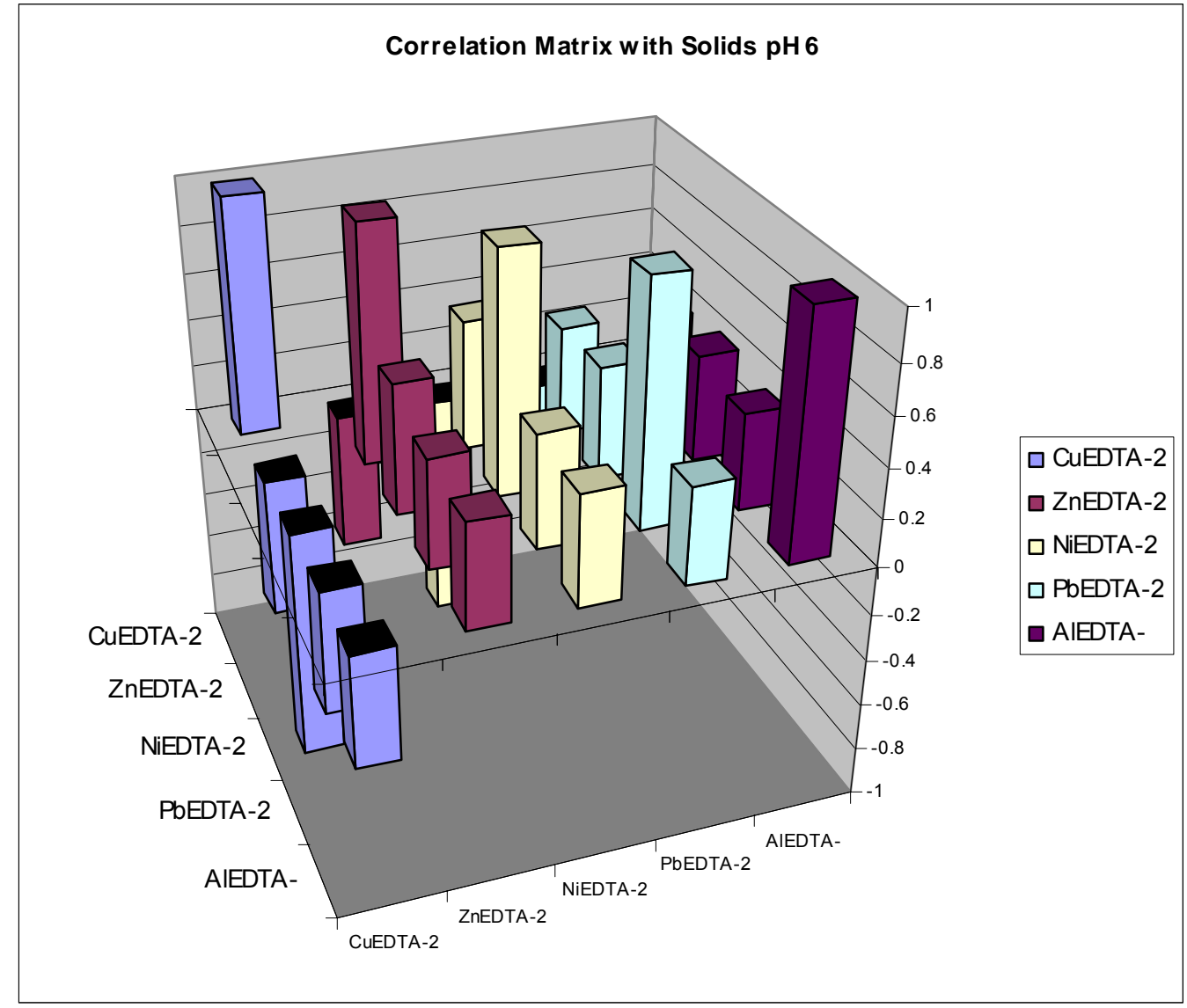

Figure S10: Correlation Matrix Series for Solids Case, pH 6 
Supporting Information-A Stochastic Regression Approach to Analyzing Thermodynamic Uncertainty in Chemical Speciation Modeling

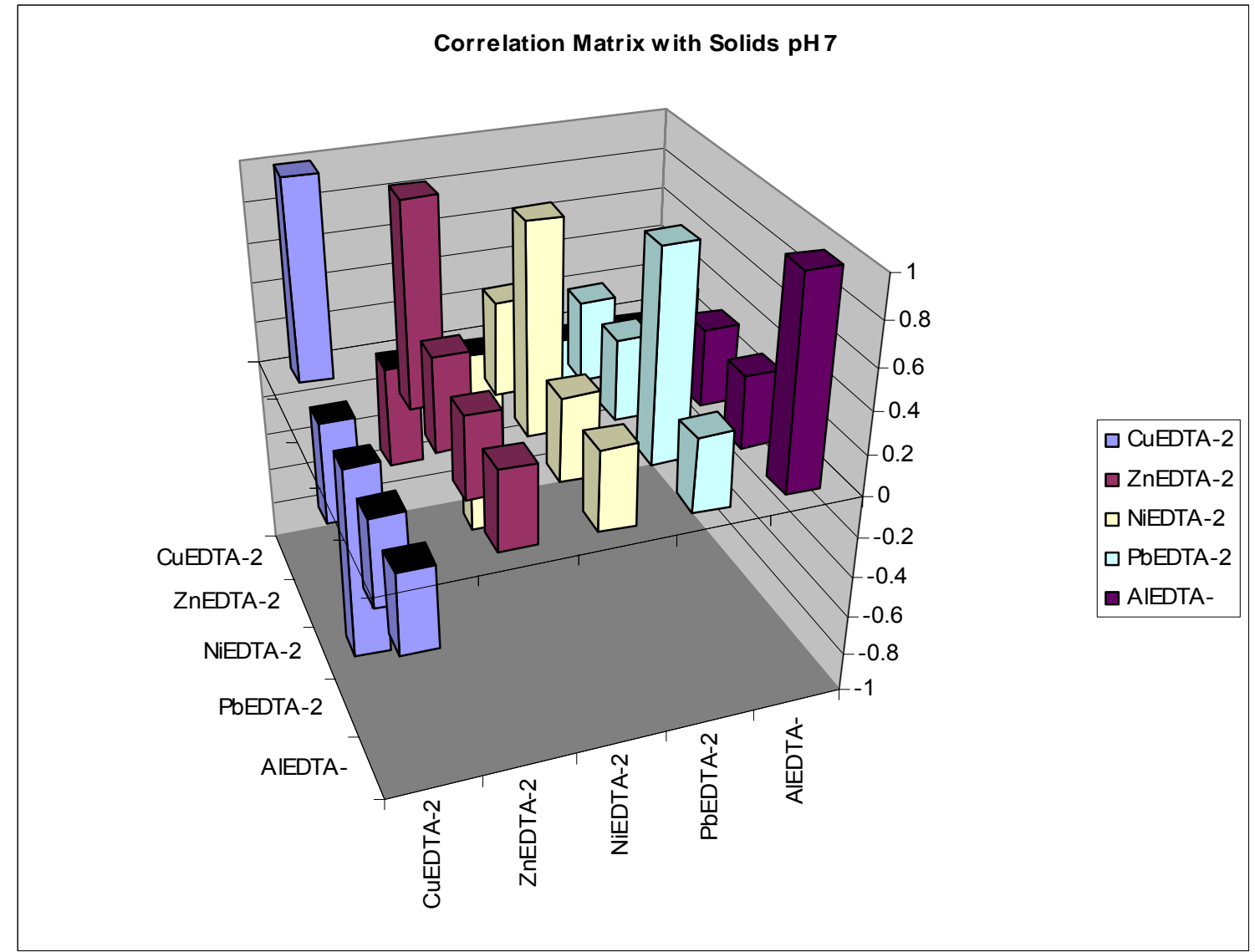

Figure S11: Correlation Matrix Series for Solids Case, pH 7 
Supporting Information-A Stochastic Regression Approach to Analyzing Thermodynamic Uncertainty in Chemical Speciation Modeling

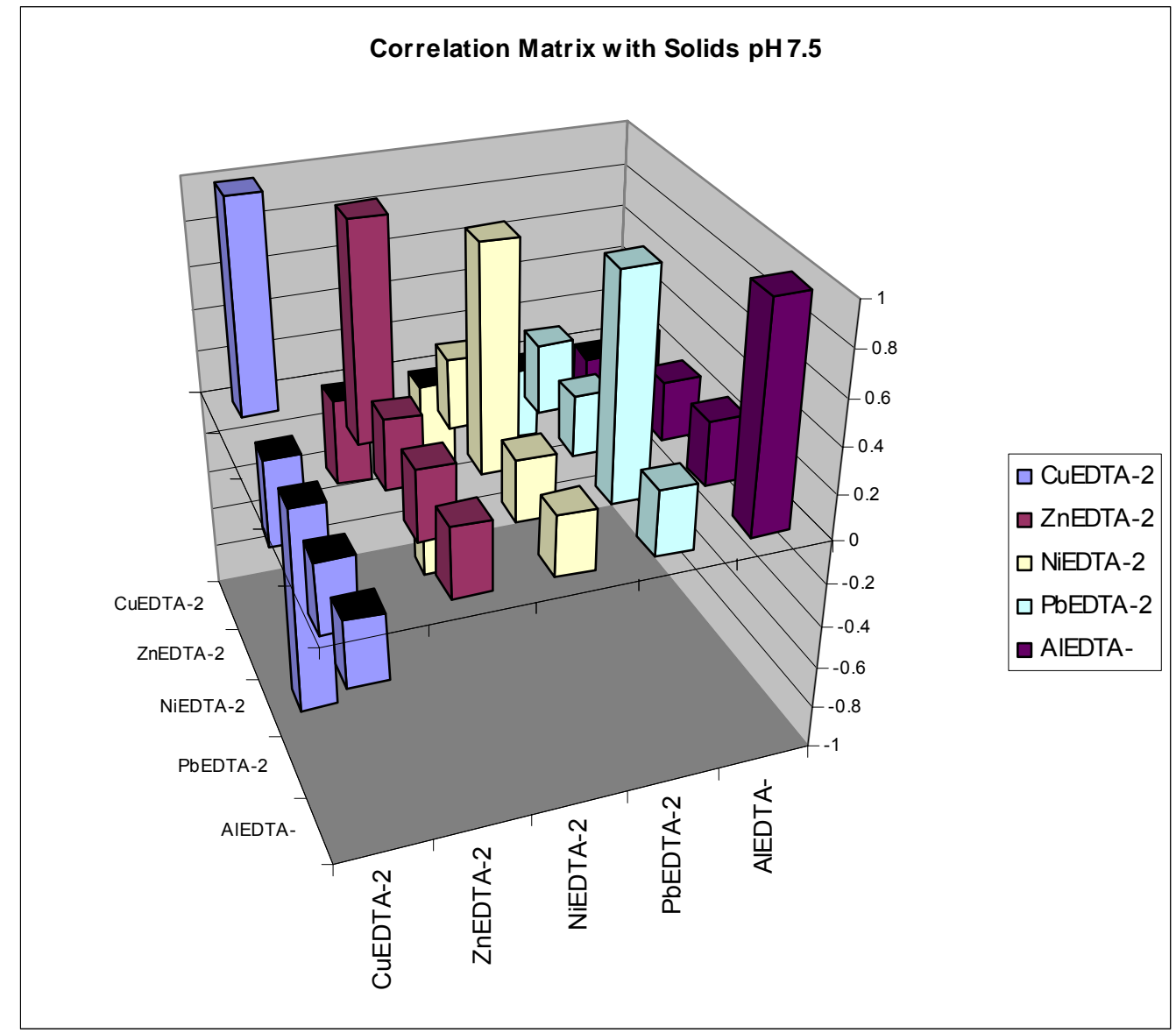

Figure S12: Correlation Matrix Series for Solids Case, pH 7.5 
Supporting Information-A Stochastic Regression Approach to Analyzing Thermodynamic Uncertainty in Chemical Speciation Modeling

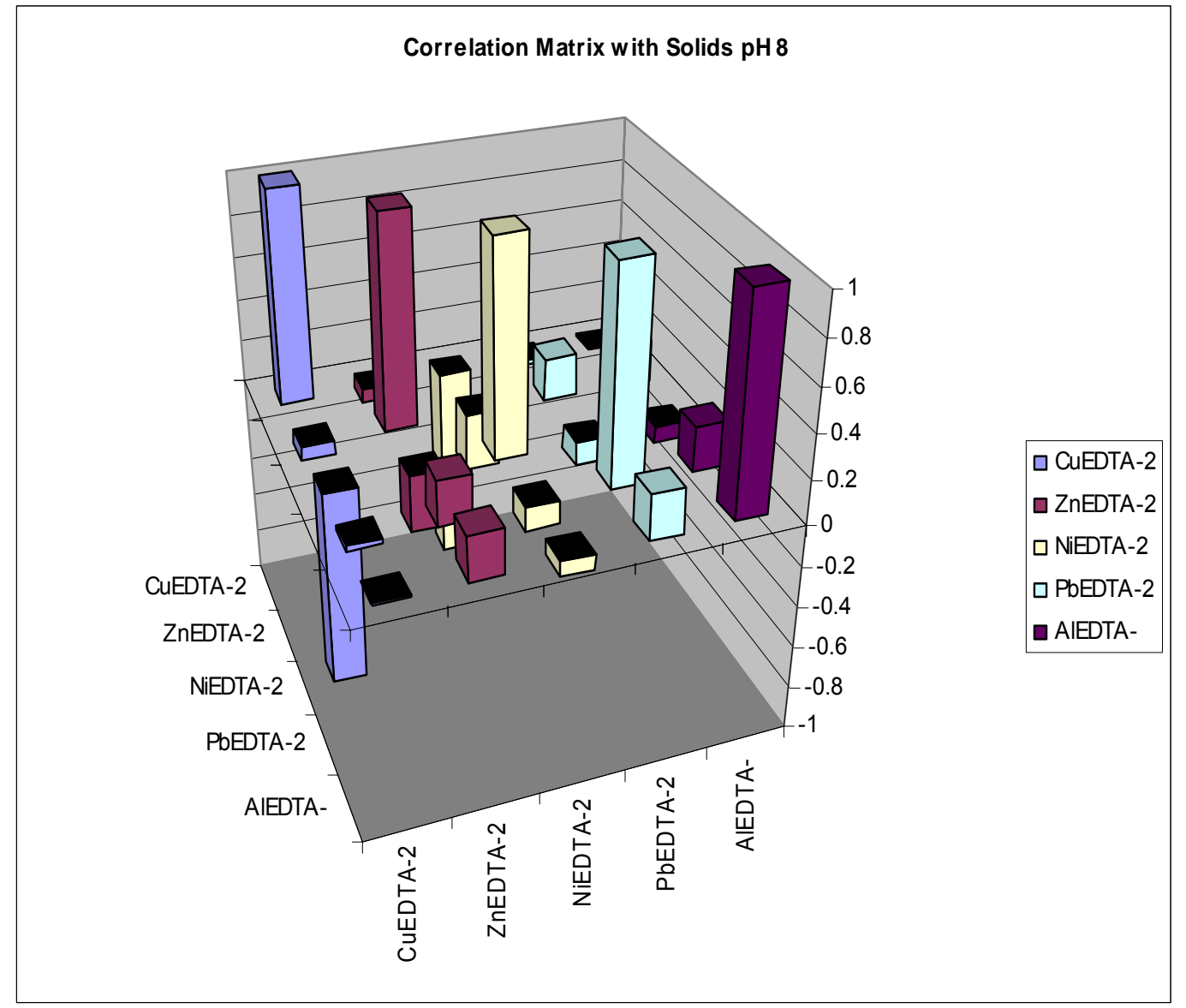

Figure S13: Correlation Matrix Series for Solids Case, pH 8 
Supporting Information-A Stochastic Regression Approach to Analyzing Thermodynamic Uncertainty in Chemical Speciation Modeling

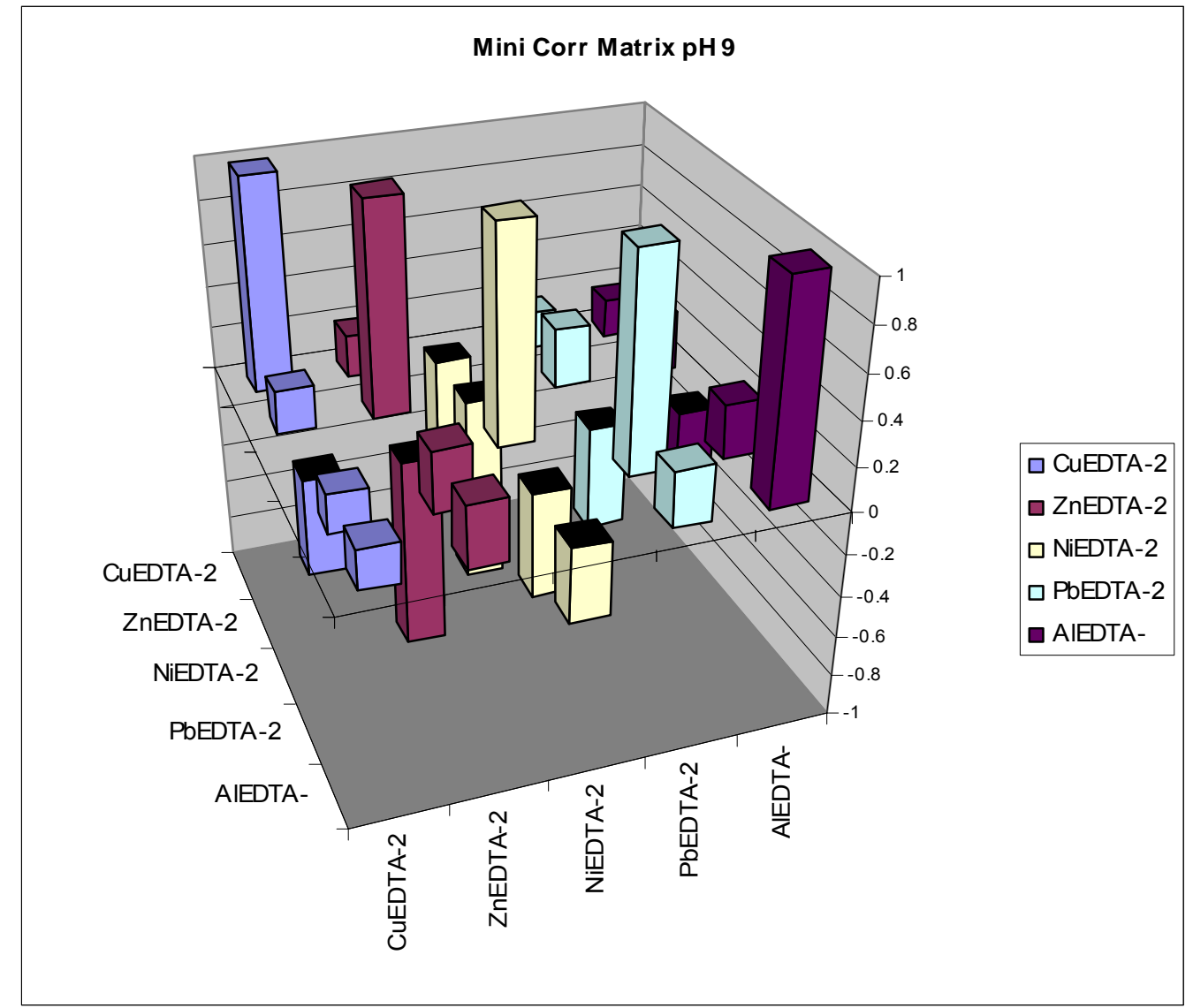

Figure S14: Correlation Matrix Series for Solids Case, pH 9 
Supporting Information-A Stochastic Regression Approach to Analyzing Thermodynamic Uncertainty in Chemical Speciation Modeling

\section{Empirical and Fitted Posterior Distributions}

As stated in the paper, several of the important output distributions from the speciation modeling portion of the work were subjected to distribution fitting in order to visually inspect the shape and skew of the distributions. Both the probability density functions (pdf's) and the cumulative distribution functions (cdf's) were utilized for this visualization, because while the pdfs provide a better visual presentation, cdf's are much easier to fit by regression. Beta distributions were used, since the values (after being transformed to percent EDTA) were constrained to be between 0 and 1 . The method of moments was used to fit the distributions with a numerical solver approach. The figures below show clearly that negative skew tended to correspond to more dominant species and positive skew corresponded with less dominant species. (compare the $\mathrm{pH}$ series to the overall system speciation shown in Figure 4 of the paper)

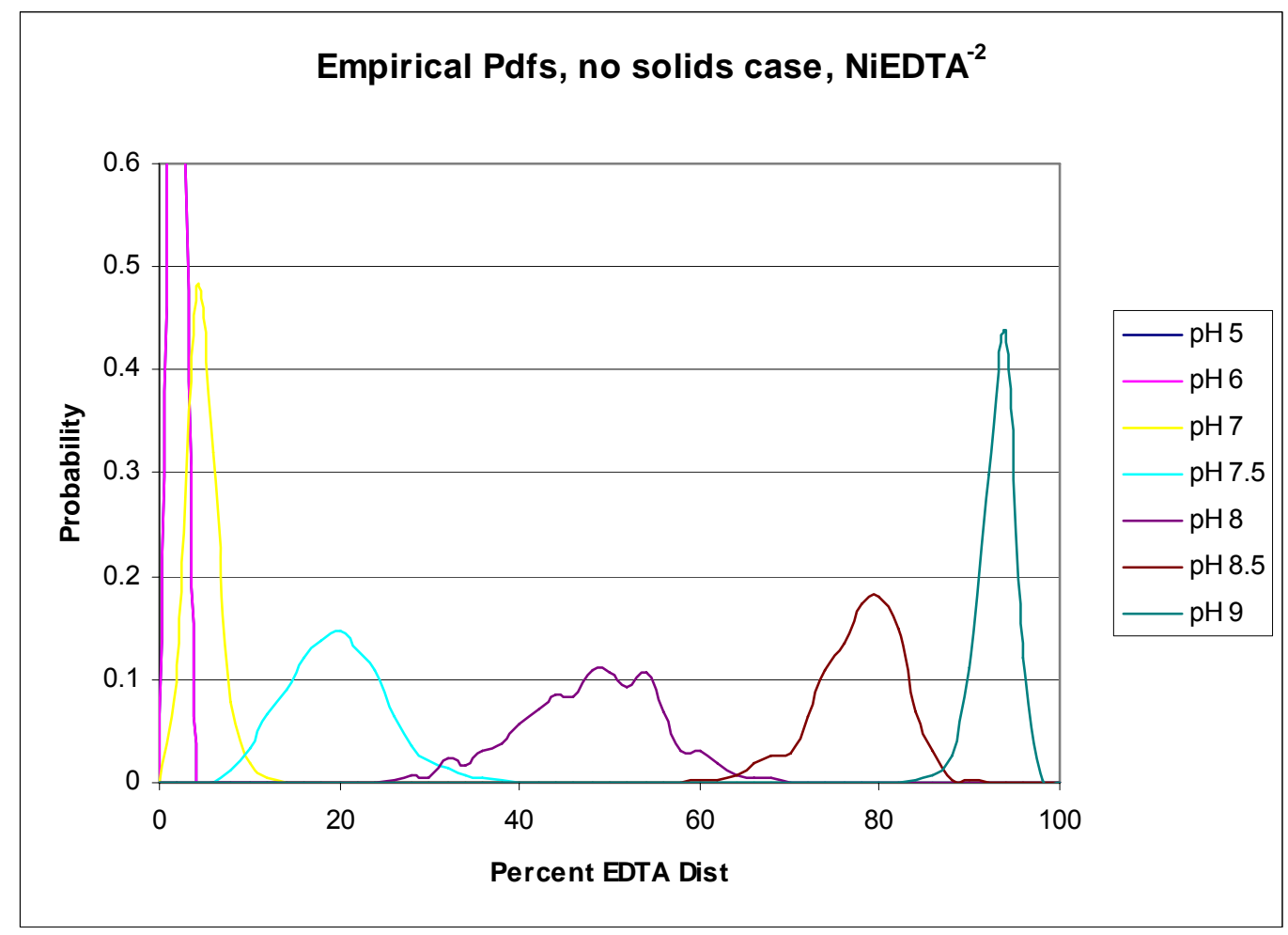

Figure S15: Empirical pdfs for NiEDTA-2 corresponding to the fitted pdfs shown in Figure 6 of the paper 
Supporting Information-A Stochastic Regression Approach to Analyzing Thermodynamic Uncertainty in Chemical Speciation Modeling

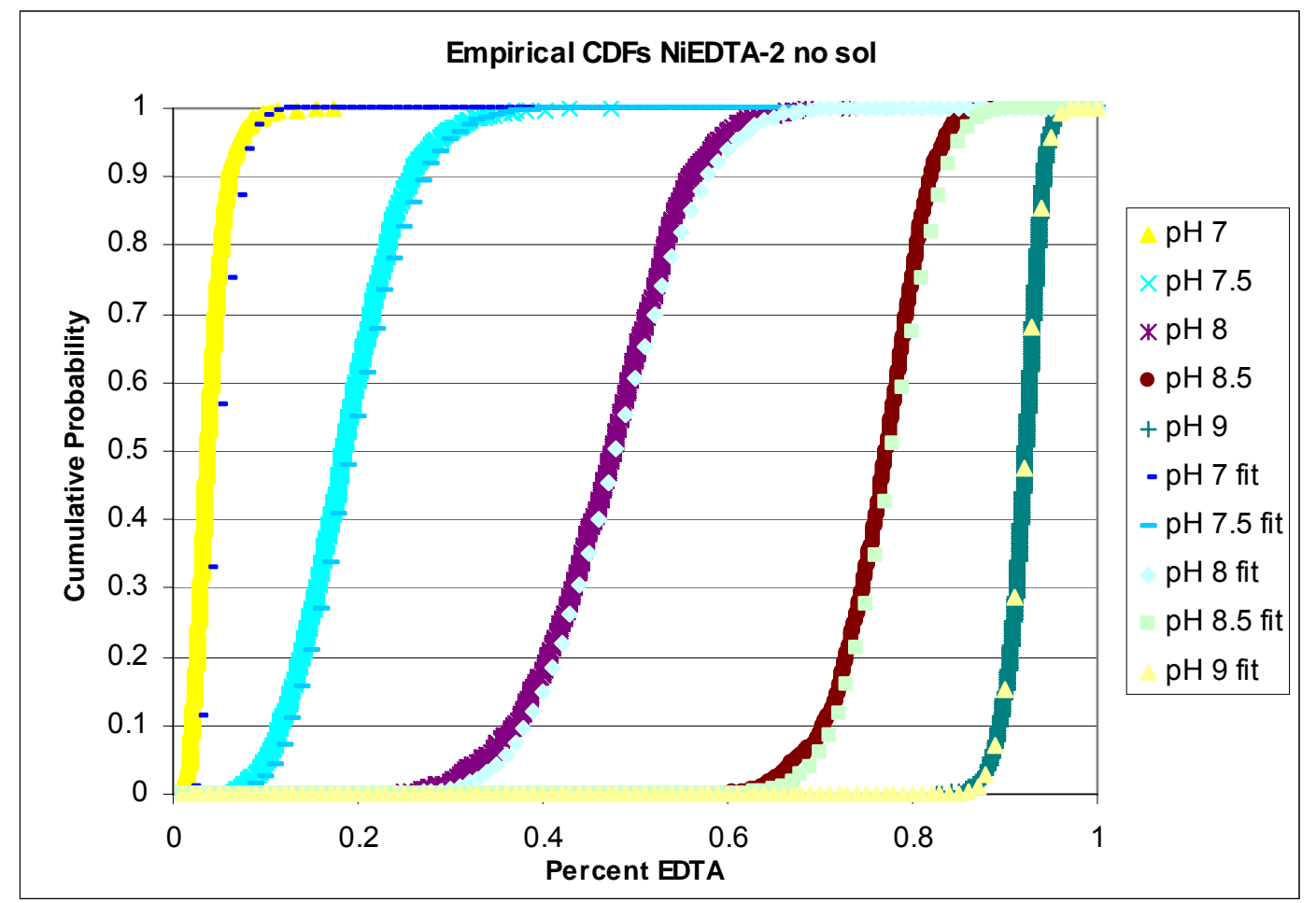

Figure S16: Comparison of empirical and fitted cumulative distribution functions for NiEDTA-2 in no solids case. It is clear that the beta distributions fit the data well.

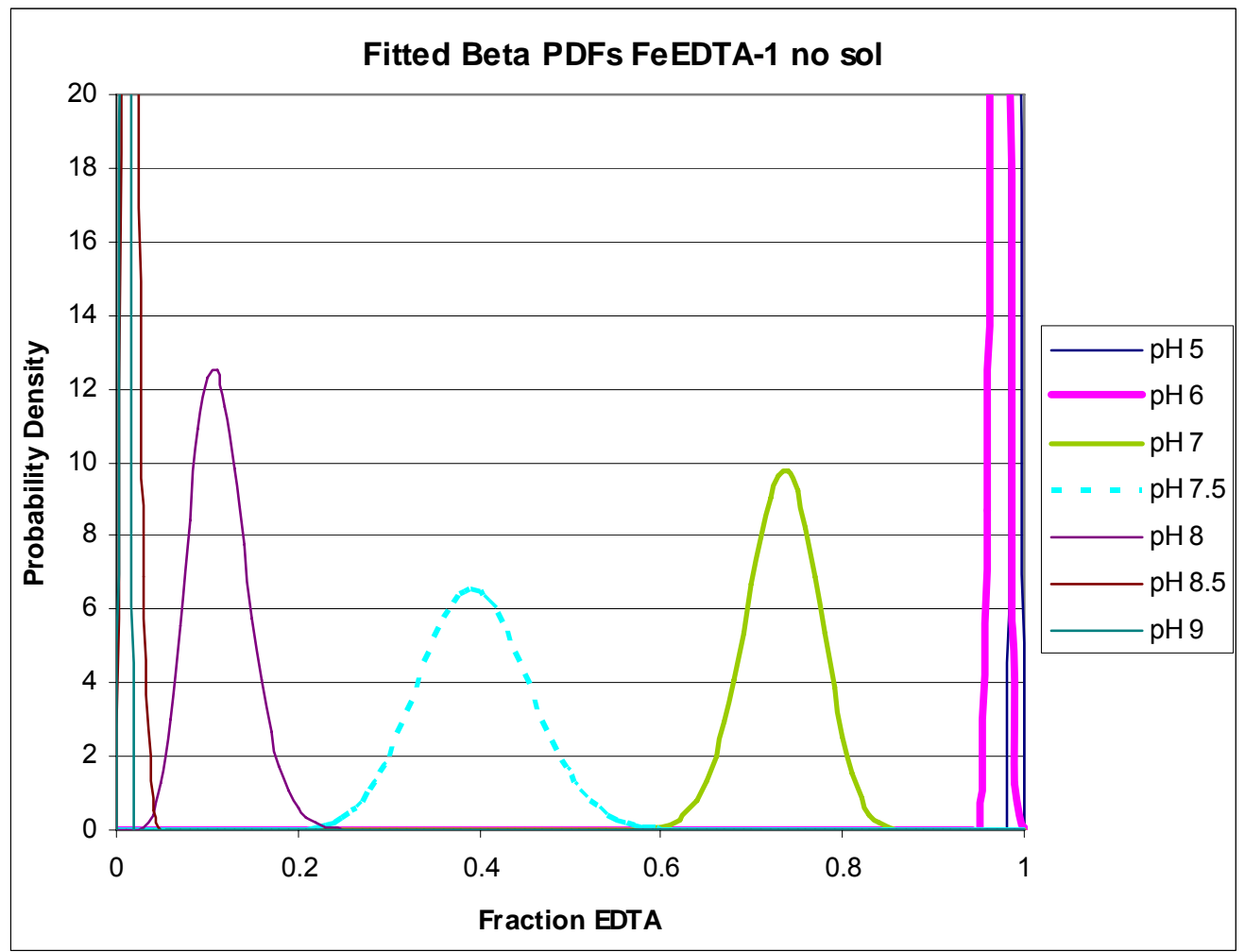

Figure S17: Fitted Beta distributions for FeEDTA-1 in no solids case 
Supporting Information-A Stochastic Regression Approach to Analyzing Thermodynamic Uncertainty in Chemical Speciation Modeling

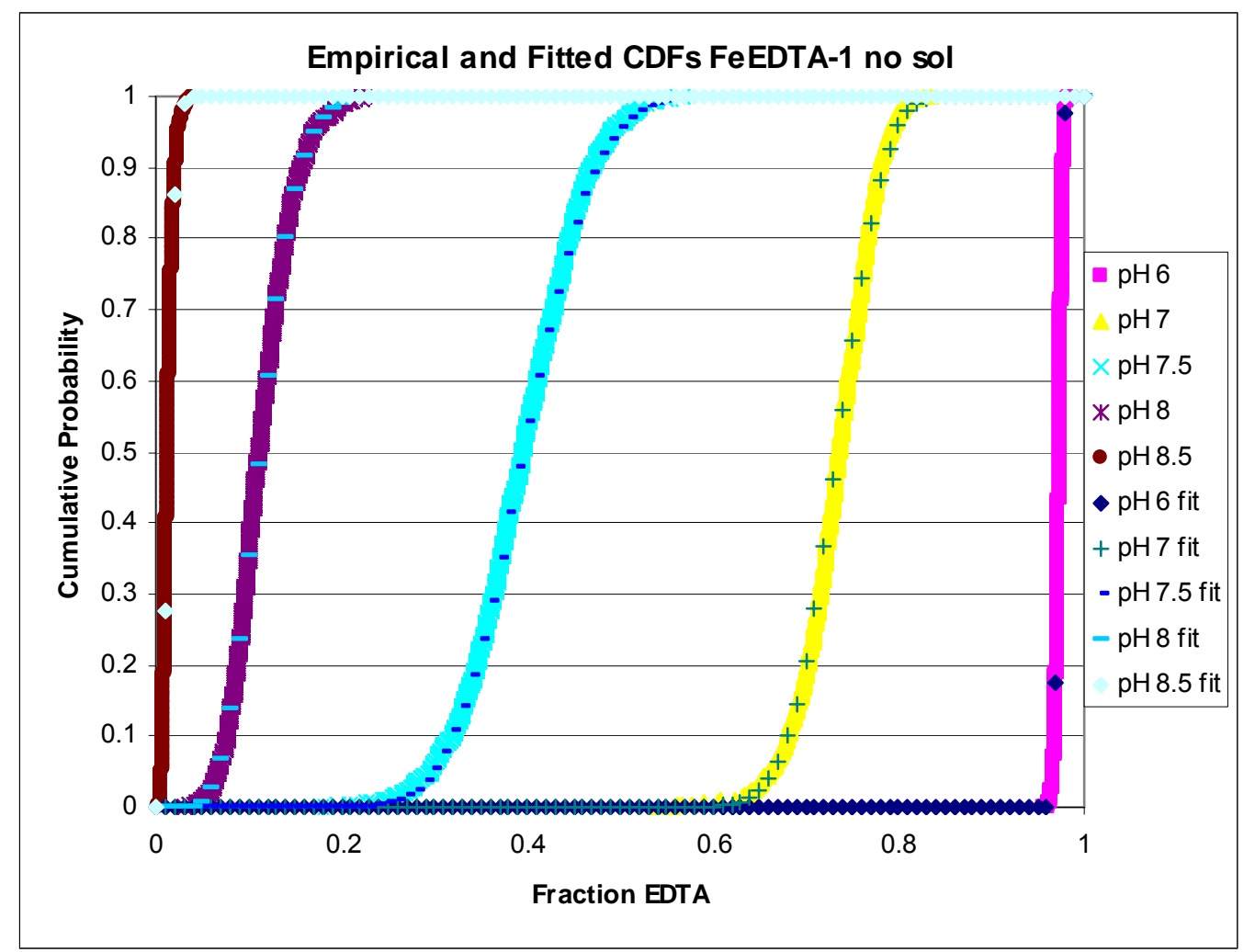

Figure S18: Comparison of empirical and fitted cumulative distribution functions for FeEDTA-1 for no solids case

\section{Literature Cited}

1. Spiegelhalter, D., Thomas, A., Best, N., and Lunn, D. WinBUGS v. 1.4. MRC Biostatistics Unit: Cambridge, UK, 2003.

2. Federal Institute for Occupational Safety and Health. Edetic Acid (EDTA) Summary Risk Assessment Report; Federal Institute for Occupational Safety and Health: Berlin, 2004 